\title{
Emergence of Distinct Electronic States in Epitaxially-Fused PbSe Quantum Dot Superlattices
}

\section{Mahmut Sami Kavrik ( $\nabla$ mkavrik@lbl.gov )}

Lawrence Berkeley National Laboratory

Jordan Hachtel

Oakridge National Laboratory https://orcid.org/0000-0002-9728-0920

\section{Wonhee Ko}

Oak Ridge National Laboratory https://orcid.org/0000-0002-6155-1485

\section{Caroline Qian}

University of California, Irvine

\section{Alex Abelson \\ University of California, Irvine}

\section{Eyup Bedirhan Unlu}

University of California San Diego

Harshil Kashyap

University of California San Diego

\section{An-Ping Li}

Oak Ridge National Laboratory https://orcid.org/0000-0003-4400-7493

Juan-Carlos Idrobo

Oak Ridge National Laboratory https://orcid.org/0000-0001-7483-9034

\section{Matt Law}

University of California, Irvine

\section{Research Article}

Keywords: quantum coupling, colloidal PbSe quantum dots, spectroscopy

Posted Date: August 26th, 2021

DOI: https://doi.org/10.21203/rs.3.rs-841852/v1

License: (9) This work is licensed under a Creative Commons Attribution 4.0 International License.

Read Full License

Additional Declarations: There is NO Competing Interest. 
Version of Record: A version of this preprint was published at Nature Communications on November 10th, 2022. See the published version at https://doi.org/10.1038/s41467-022-33955-w. 


\section{Emergence of Distinct Electronic States in Epitaxially-Fused PbSe Quantum Dot Superlattices}

Mahmut S. Kavrik ${ }^{1,2^{*}}$, Jordan A. Hachtel ${ }^{3^{*}}$, Wonhee $\mathrm{Ko}^{3^{*}}$, Caroline Qian ${ }^{4}$, Alex Abelson ${ }^{5}$, Eyup

B. Unlu ${ }^{2}$, Harshil Kashyap², An-Ping Li ${ }^{3}$, Juan C. Idrobo ${ }^{3}$, Matt Law ${ }^{4,5,6}$

${ }^{1}$ Materials Sciences Division, Lawrence Berkeley National Laboratory, Berkeley, CA

${ }^{2}$ Department of Materials Science and Engineering, University of California, San Diego, CA

${ }^{3}$ Center for Nanophase Materials Sciences, Oak Ridge National Laboratory, Oak Ridge, TN

${ }^{4}$ Department of Chemical and Biomolecular Engineering, University of California, Irvine, CA

${ }^{5}$ Department of Materials Science and Engineering, University of California, Irvine, CA

${ }^{6}$ Department of Chemistry, University of California, Irvine, CA 92697

Corresponding authors: Mahmut S. Kavrik, (mkavrik@lbl.gov); Jordan A. Hachtel (hachtelja@ornl.gov); Matt Law, (matt.law@uci.edu)

*Indicated authors contributed equally.

Notice: This manuscript has been authored by UT-Battelle, LLC, under Contract No. DEAC0500OR22725 with the U.S. Department of Energy. The United States Government retains and the publisher, by accepting the article for publication, acknowledges that the United States Government retains a non-exclusive, paid-up, irrevocable, world-wide license to publish or reproduce the published form of this manuscript, or allow others to do so, for the United States Government purposes. The Department of Energy will provide public access to these results of federally sponsored research in accordance with the DOE Public Access Plan (http://energy.gov/downloads/doe-public-access-plan). 


\begin{abstract}
Quantum coupling in arrayed nanostructures may induce novel mesoscale properties such as electronic minibands that may lead to applications including high efficiency solar cells. Colloidal PbSe quantum dots (QDs) can self-assemble into epitaxially-fused superlattices (epi-SLs), making them a promising material system to study collective phenomena. In the present study, the presence of distinct local electronic states induced by crystalline necks connecting individual PbSe QDs is documented by several techniques that leads to modulation of the band gap energy across the epiSL. The energy band gap measured by multi-probe scanning tunneling spectroscopy (STS) shows variation from $0.7 \mathrm{eV}$ at the center of the QDs to $1.1 \mathrm{eV}$ at their necks. Complementary monochromated electron energy-loss spectroscopy (EELS) measurements reveal the presence of distinct electronic states from necks in the epitaxial superlattices (epi-SL), confirming the STS measurements and demonstrating band gap modulation in spectral mapping. It is hypothesized that these new electronic states are induced by quantum confinement of carriers in the necks between the QDs, redefining the energy landscape of the PbSe QD epi-SL.
\end{abstract}




\section{Introduction}

The electronic and photonic properties of colloidal semiconductor quantum dots (QDs) can be controlled by tailoring their shape and size $\mathrm{e}^{1,2}$. Their electronic structure differs from that of macroscopic crystals due to confinement of the electron and hole wave functions within the QD, resulting in discrete "atom-like" energy states rather than continuous bands ${ }^{3,4}$. In principle, the energy landscape of QD systems can be deliberately changed via epitaxially fusing QDs into spatially- and energetically-ordered superlattices (SL), strengthening the electronic coupling between QDs and inducing carrier delocalization throughout the entire superlattice. The inter-QD coupling in QD superlattices has been theorized to promote the emergence of electronic minibands that yield high carrier mobility, topological states, and Bloch oscillations ${ }^{5-7}$. Recent studies have focused on understanding the self-assembly and ligand exchange processes needed to synthesize highly-ordered epitaxially-fused QD superlattices (epi-SLs) ${ }^{8}$. Among colloidal nanocrystals, PbSe QDs are promising for optoelectronic applications because their large exciton Bohr radius results in strong quantum confinement and wavefunction overlap between neighboring QDs ${ }^{9}$. Recently, three-dimensional PbSe QD epi-SLs with micron-sized grains have been demonstrated as a promising system for studying mesoscale physics ${ }^{10}$. However, it is still unknown how epitaxial fusion modifies the local electronic structure of the coupled QDs, which is crucial to understanding and controlling their mesoscopic behavior.

In this work, the local electronic structure of PbSe QD epi-SLs is probed using a combination of scanning tunneling microscopy/spectroscopy (STM/STS) and monochromated aberrationcorrected scanning transmission electron microscopy/electron energy loss spectroscopy (STEMEELS). A long-range periodic order between epitaxially fused QDs is observed, in agreement with previous reports $^{11}$. The STM-STS measurements revealed an average QD band gap of $0.7 \mathrm{eV}$, 
close to the optical band gap of the colloidal QD dispersion, while the smaller epitaxial connections (necks) have a band gap of $1.1 \mathrm{eV}$, in good agreement with previous studies on the relationship between band gap and PbSe QD diameter ${ }^{12}$. Complementary EELS measurements show that the epi-SL has an onset of energy loss at $1.1 \mathrm{eV}$ and unique spectral features between $1.1 \mathrm{eV}$ and $\sim 4$ $\mathrm{eV}$ not observed in isolated QDs. The $1.1 \mathrm{eV}$ band gap energy and low-energy interband transitions are consistent with electronic states induced by quantum confinement within the epitaxial necks between QDs suggesting the emergence of distinct electronic states within the necks that change the energy landscape of the epi-SLs. 


\section{Structure of the PbSe QD Epi-SL Samples}

Epi-SLs formed by QDs fused to each other by oriented attachment were fabricated by selfassembly and ligand exchange on the surface of liquid ethylene glycol ${ }^{10}$. In this work, the electronic structure of (i) a multilayer (3D) epi-SL thin-film was investigated with STM/STS and (ii) a similarly-prepared monolayer (2D) epi-SL sample was studied with EELS. A multilayer epiSLs were employed for STM because they have larger, more uniform SL grains and are better suited to two-probe STS experiments than QD monolayers, which have many lateral discontinuities to current flow. EELS measurements were performed on monolayer epi-SLs in order to simplify the imaging and image analysis. Although the fabrication and the structure of the 2D epi-SL differs from those of the 3D epi-SL, the building block - QD/neck/QD - of both the 2D and 3D epi-SLs is similar for both samples.

The 3D epi-SL sample was fabricated by drop casting a dispersion of oleate-capped PbSe QDs (6.5 nm diameter) in hexanes onto the surface of ethylene glycol (EG), allowing the hexanes to evaporate while the QDs self-assemble on the liquid surface, and injecting a solution of 1,2ethylenediamine (EDA) into the EG to trigger oleate removal and epitaxial fusion of the QDs. This procedure results in a polycrystalline 3D epi-SL with a distorted simple cubic unit cell. Details of the sample fabrication and structural analysis can be found elsewhere ${ }^{10,11}$. The 2D epi-SLs were made by drop-casting the oleate-capped $\mathrm{PbSe} \mathrm{QDs}$ in toluene onto a $\mathrm{SiO}_{2}$ solid substrate and treating the monolayer with ammonium thiocyanate to remove oleate and trigger epi-fusion (see Methods).

Atomic-resolution high-angle annular dark field (HAADF) STEM was employed to investigate the structure of the monolayer epi-SL samples. Figure 1a shows a representative largearea HAADF image of an epi-SL, which consists of a QD monolayer with a small bilayer region 
(high contrast sliver). Various structural defects are visible in this image, including missing QDs (vacancies), missing necks, and misoriented QDs, consistent with previous reports on these systems ${ }^{11}$. The QDs are connected to each other in most regions of the sample (i.e., few isolated QDs) with varying degrees of epitaxial necking, resulting in a mesoscopic slightly oblique lattice. Examples of QDs that are isolated (zero nearest neighbors (NNs)), incompletely-connected $(<4$ NNs), and fully-connected (4 NNs) are shown in Figs. 1b-1d. Atomic-resolution imaging shows that the QDs are monocrystalline, in epitaxial registry with their neighbors, and primarily fuse along the PbSe $\{100\}$ facets. Representative size nonuniformity of the QDs and necks in the epiSL is highlighted in the magnified image in Fig. 1e.

Fig. If is a histogram of the size distribution of the QDs and necks in Fig. 1e. Details about the algorithm to determine the QD diameter are presented in Fig. S1 of the Supplementary Information. The red and blue highlighted regions in Fig. 1e denote the measured diameters of the QDs and necks, respectively, obtained from this analysis. The mean QD (among 361 QDs) diameters and neck diameters (among 531 necks) are $5.5 \mathrm{~nm}$ and $3.5 \mathrm{~nm}$, with standard deviations of $0.5 \mathrm{~nm}$ and $0.6 \mathrm{~nm}$, respectively, consistent with previous reports ${ }^{10,11}$. 

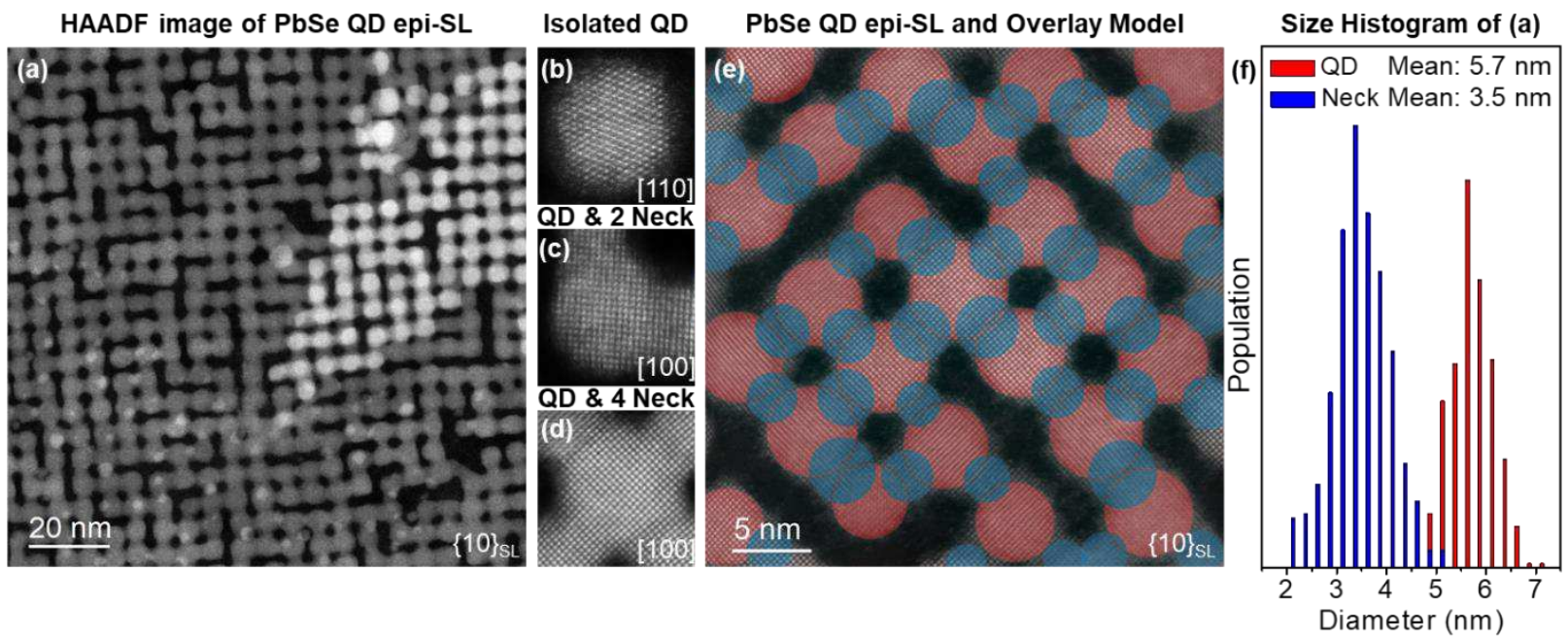

Figure 1. Structural analysis of the monolayer PbSe QD epi-SL. (a) HAADF-STEM image of an epi-SL showing various structural defects, including missing QDs and necks. (b-d) Atomicresolution HAADF reference images of (b) an isolated QD, (c) a QD with two necks, and (d) a QD with four necks demonstrating the crystallinity of the QDs and the epitaxial nature of the necks. (e) QD and neck diameters measured from a HAADF-STEM image. Red and blue circles denote QD and neck diameters, respectively. (f) Histogram of QD and neck diameters from the image. Average QD diameter: $5.7 \pm 0.5 \mathrm{~nm}$; average neck diameter: $3.5 \pm 0.6 \mathrm{~nm}$. 


\section{Investigating the Local Electronic Structure with STM/STS}

The influence of inter-QD necking on the local electronic structure of the epi-SL was investigated using STM/STS. These experiments measure the differential tunneling conductance, $\mathrm{d} I / \mathrm{d} V$, which is proportional to the local density of states (LDOS); therefore, the local electronic structure can be investigated directly ${ }^{13}$. However, maintaining a stable tunneling condition is highly challenging for the conventional single-probe STM configuration in which the substrate under the epi-SL is biased and the STM tip scans across the QDs. The instability results from the electrical resistance between the epi-SL and substrate being moderated by residual surface ligands and band offsets that result in a large resistance that obstructs the tunneling current ${ }^{14,15}$. Desorption of the surface ligands by high-temperature annealing and functionalization of the conductive substrates with linkers such as dithiols is problematic on account of the heat- and air-sensitive $\mathrm{PbSe}_{\mathrm{QDs}}{ }^{14}$. To minimize the electrical resistance between epi-SL and substrate, sub-monolayer of epi-SL can be deposited on metallic surfaces and observed by STM/STS, but the interaction between the QDs and substrate strongly affects the electronic band of QDs. ${ }^{16,17}$

In this work, this issue was resolved by employing a two-probe STM configuration: one STM probe was placed in the tunneling position with a feedback loop to scan the sample, and the other in contact with a nearby part of the film to bias the epi-SL and collect the tunneling current from the first probe (the geometry is shown schematically in Fig. 2a and Fig. S5) ${ }^{18,19}$. This configuration enables effective STS measurements of the epi-SL in the shell-tunneling regime (no carrier accumulation) since the tunneling current across the epi-SL bypasses the epi-SL-substrate barrier $^{20}$. Therefore, the spectral features correspond to the energy levels of the epi-SL directly.

Fig. 2b shows an STM image acquired on a (100)SL-oriented 3D epi-SL (6-7 QD layers, 40 nm thick), demonstrating the capability of resolving individual QDs and necks directly from 
the tunneling current. This STM image was used as a reference to measure the LDOS at the center of the QDs and the midpoint of the necks. The influence of necking on the LDOS is observed by comparing $\mathrm{d} I / \mathrm{d} V$ spectra of a QD (R1-red circle) and a neck (R2-blue circle), which are shown in Fig. 2c. Each spectrum is an average of ten spectrums taken in the same location. In the spectrum of the QD (R1), the tunneling current diminishes to noise level between $-0.35 \mathrm{eV}$ and $0.35 \mathrm{eV}$, indicating an electronic band gap of $0.7 \mathrm{eV}$, while the STS from a neck (R2) shows a $1.1 \mathrm{eV}$ band gap, distinctively larger than that of the QD. The electronic band gap of a QD is inversely proportional to its diameter, and this relationship has been determined for PbSe QDs by Moreels et. al., as shown in Fig. $2 \mathrm{~d}^{12}$. By overlaying the average QD and neck diameter values that were established in the histogram shown in Fig. 1f, a band gap value of $0.68 \mathrm{eV}$ is observed for the QDs and $1.05 \mathrm{eV}$ is observed for the necks according to Fig. $2 \mathrm{~d}$. These values match well with the band gaps of both the QDs and necks in the STS measurements from Fig. 2c. The increase of the band gap on necks relative to QDs was consistently observed across the epi-SL surfaces in various conditions including different surface orientation of the epi-SL and the surface treatments (Fig. S4 and S5)

While the inverse correlation between QD band gap and their average diameters is expected, observing a similar relation for the necks is notable. This suggests the confinement of carriers and emergence of new electronic states in the necks similar to those present in the QDs. More critically, the tunneling spectra both in the QDs and the necks do not show discrete energy levels like those of a single isolated QD, as reported previously ${ }^{13}$, but rather a smoothly increasing LDOS above the band edges. The absence of resonance peaks near the band edges may result from quantum coupling or other causes such as thermal broadening, electron-phonon coupling, or intervalley coupling, all of which may broaden the $\operatorname{LDOS}^{21-23}$. 
To gain statistical evidence of the distinct band gaps between QDs and necks, we performed STS measurements at equally spaced grid positions over the entire $35 \mathrm{~nm} \times 35 \mathrm{~nm}$ area shown in Fig. 2b. The band gap at each of the 81 nominal probe positions is tabulated in the histogram in Fig. 2e. The histogram shows a distribution of band gaps between $0.6 \mathrm{eV}$ and $1.6 \mathrm{eV}$ with sharp peaks at $0.7 \mathrm{eV}$ and $1.1 \mathrm{eV}$, in agreement with the values of the QD and neck band gaps in Fig. 2d, respectively. It should be noted that it is not possible to rigorously associate the band gaps from the STS grid with the probe position due to the spatial drift of the tip and the tip convolution caused by its comparably large radius of curvature (the typical tip radius of curvature is $20-100 \mathrm{~nm}$, significantly larger than the feature size of $3-7 \mathrm{~nm})^{24,25}$. Furthermore, there is no distinct feature in Fig $2 \mathrm{~d}$ from the hollows because when the probe goes down into the hollow, the tunneling occurs between the side of the STS probe and the side of the QDs. From this histogram, the mean value of the band gap of QD: $0.72 \pm 0.05$ and neck: $1.2 \mathrm{eV} \pm 0.2$ was found by considering neck diameter being smaller than $4 \mathrm{~nm}$. Nonetheless, the histogram peaks at the QD and neck band gaps is consistent with some significant fraction of the film having a band gap of $1.1 \mathrm{eV}$, which we attribute to the necks (population of the necks are twice the size of the QD as seen in Fig. 1). This corroborates the hypothesis that the necks act as more confined regions of the QD film and induce new states into the electronic structure of the epi-SL. 

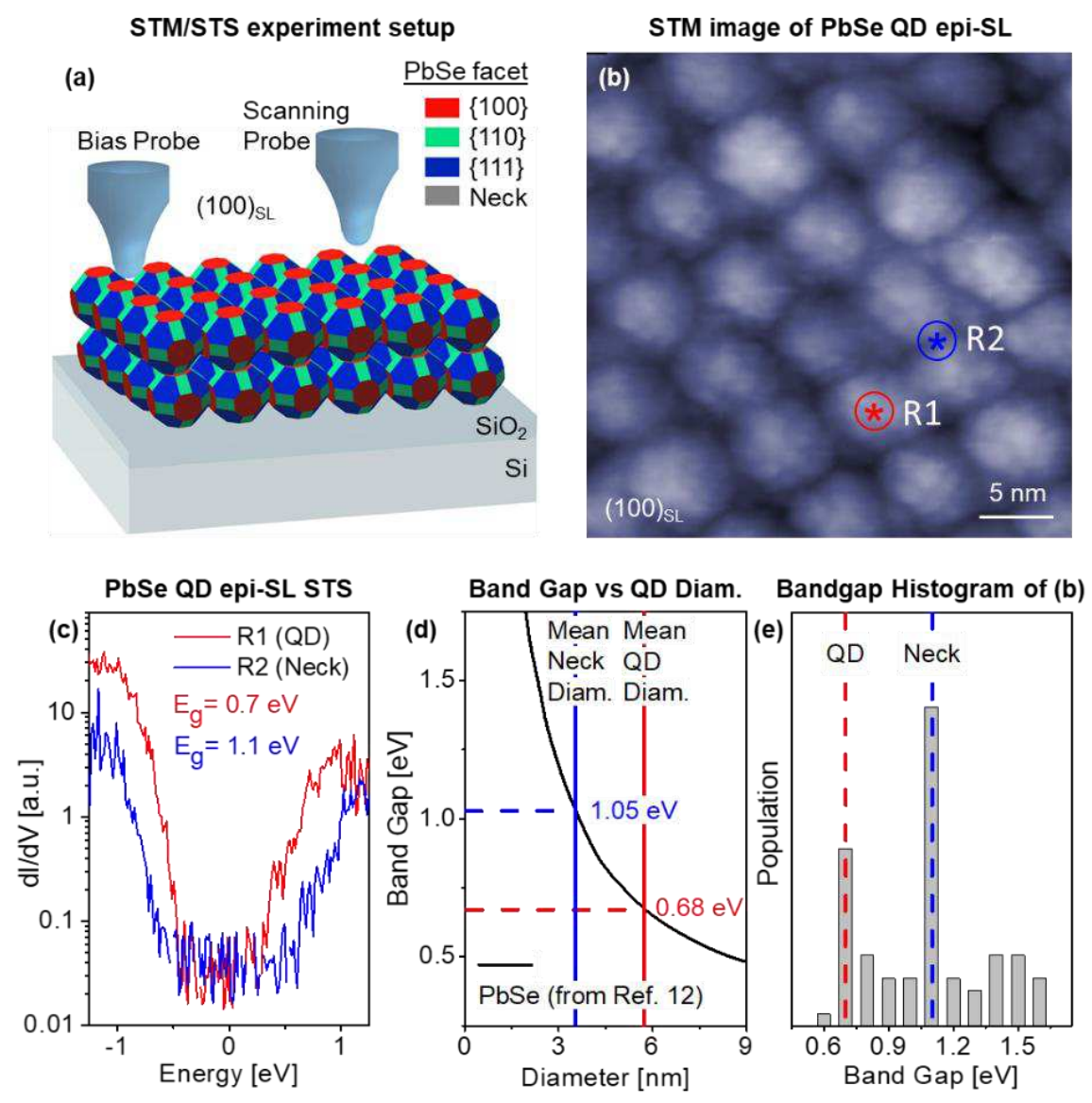

Figure 2. Electronic structure of multilayer epi-SLs probed by STM/STS. (a) Cartoon

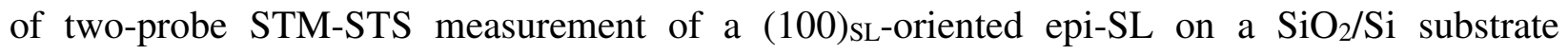
(illustration is not to scale). We note that the radius of curvature of the STM tip (20-100 nm) is larger than the size of the necks and QDs (3-7 nm). Tunneling current was measured between a bias probe in soft contact with the epi-SL and a scanning probe that was kept in a tunneling position by the feedback loop. (b) A representative STM image of an (100)SL-oriented epi-SL grain $(V=$ $3 \mathrm{~V}, I=1 \mathrm{pA}$ ). Red and blue symbols denote nominal probe locations for STS in (c). (c) Representative $\mathrm{d} I / \mathrm{d} V$ spectra acquired from the QD center (red circle in (b)) and neck (blue circle in (b)) adjoining two QDs. Band gaps of $0.7 \mathrm{eV}$ for the QD and $1.1 \mathrm{eV}$ for the neck were extracted from the spectra. (d) Dependence of QD band gap on diameter from Ref 12. (e) Histogram of the band gap values obtained from 81 STS locations in (b). The histogram shows peaks at $0.7 \mathrm{eV}$ and $1.1 \mathrm{eV}$, which are hypothesized to correspond to the expected band gaps of the QDs and necks, respectively. 


\section{Investigating Local Electronic Structure with EELS}

The electronic structure of QDs can be investigated using EELS, which has been previously

performed on isolated $\mathrm{PbS}$ QDs using $\mathrm{EELS}^{26,27}$. Here, the local electronic structure of a monolayer PbSe QD epi-SL was investigated with EELS in a monochromated aberration-corrected $\mathrm{STEM}^{28}$. In Fig. 3, a hyperspectral EELS analysis was performed of a region possessing a single isolated QD (no necking, $0 \mathrm{NNs}$ ) next to a section of monolayer epi-SL (with necking and >0 NNs). The region analyzed with EELS is shown with green rectangle in HAADF image in Fig. 3. An EEL hyperspectral dataset called a spectrum image (SI), meaning an EEL spectrum is acquired at each probe position in a $30 \times 95$ grid of pixels to create a three-dimensional dataset with two spatial dimensions and one spectral dimension. The HAADF in Fig. 3b is simultaneously-acquired with the SI allowing to highlight specific pixels based on the structure and directly plot their representative spectra. The pixels selected from the spectra for supporting substrate $\left(\mathrm{SiO}_{2}\right)$, the isolated dot (QD), and the epitaxially connected structure (epi-SL) are shown in Fig. 3c.

In all three spectra, the signal at ultralow energies is dominated by the tail of the elastic scattering or zero-loss peak (ZLP). The tail is a function of the instrumental condition, not the sample, and is therefore nearly identical in all three spectra. Also common to all three spectra is the band edge of the supporting $\mathrm{SiO}_{2}$ substrate $(>8.5 \mathrm{eV})$, which is expected since the substrate is uniformly present everywhere in the region. Here, it is critical to note that EELS samples the electronic structure by measuring the energy-loss associated with transitions from the valence band to the conduction band making it analogous to the joint density of states (JDOS) ${ }^{28}$. Therefore, excitations such as a band-gap do not manifest as a peak at the band-gap instead they manifest as an onset of EEL intensity at the band-gap energy. Thus, the peak EEL intensity associated with the $\mathrm{SiO}_{2}$ bandgap is at $10.5 \mathrm{eV}$, even though the bandgap itself is at $8.5 \mathrm{eV}$. Examining the two 
spectra from the $\mathrm{PbSe}$ QDs, with respect to the $\mathrm{SiO}_{2}$ spectra, an onset of intensity at low energies (close to the isolated QD band gap of $0.7 \mathrm{eV}$ ) are seen. However, the epi-SL spectrum has an additional onset of increased intensity with respect to the QD and this onset corresponds closely to the nominal bandgap of the QD necks $(1.1 \mathrm{eV})$.

The effect can be more clearly visualized with difference spectra to remove the influence of elastic scattering and the substrate, these are shown in Fig. 3d. The "QD-SiO" subtracting the representative spectra from the isolated QD from the substrate spectrum) represents the electronic structure of the individual $\mathrm{PbSe} \mathrm{QD}$, and here the difference spectrum becomes positive only at $0.7 \mathrm{eV}$. The "SL-QD" (subtracting the epi-SL spectra from the isolated QD spectrum) represents the electronic structure modifications to the PbSe induced by the epitaxial connectivity. Here, the difference spectra for the SL-QD hasa new onset of intensity, above the $\mathrm{PbSe} \mathrm{QD}$, starting at $1.1 \mathrm{eV}$ is clearly seen. Critically, the EELS observations of an onset of intensity at $0.7 \mathrm{eV}$ in the isolated $\mathrm{QD}$ and the onset of intensity at $1.1 \mathrm{eV}$ in the epi-SL are consistent with the STS experiments in Fig. 2 for the band gaps of the QDs and their necks.

To visualize the spatial localization of these effects, and compare them across multiple datasets, a component separation technique called non-negative matrix factorization (NMF) was utilized. NMF decomposes the spectrum images (SIs) into a set number of components, each with a spectral endmember and a spatial abundance map, where the linear combination of each endmember at the intensity of the abundance maps reconstructs the original dataset. For simple systems, the spectral endmembers correspond directly to spectral phenomena in the system with different localizations, and the spatial abundance maps provide a visualization of the location and intensity where these phenomena occur. 

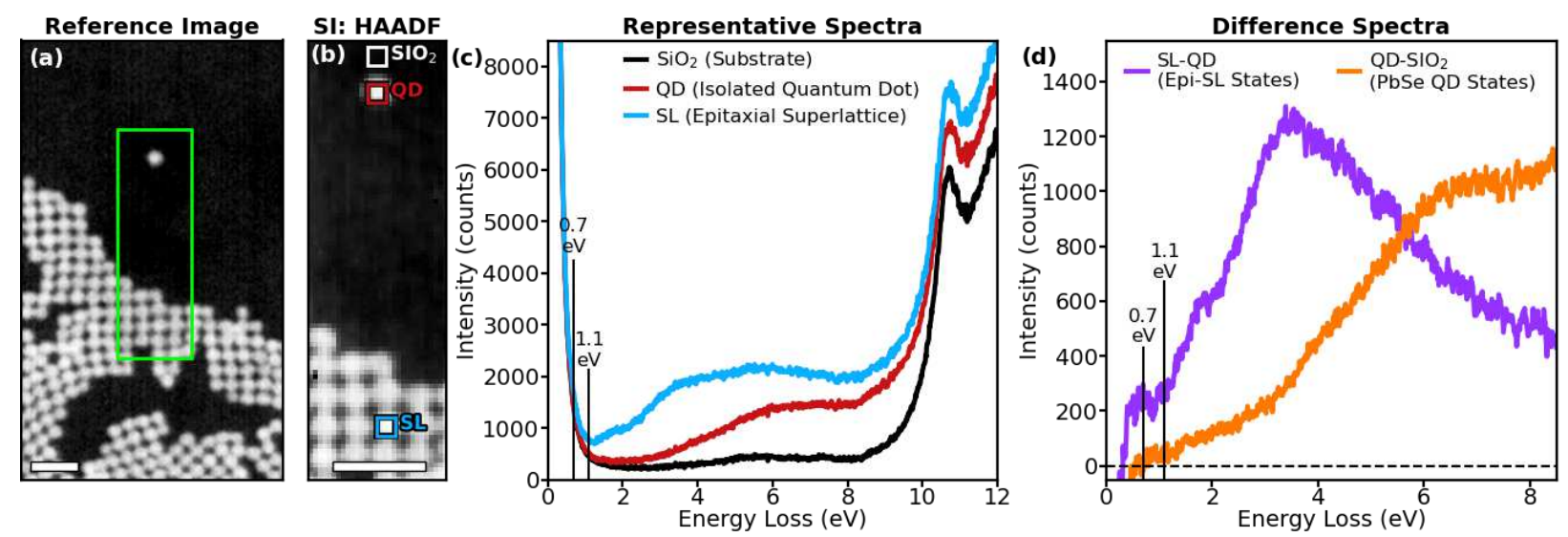

Fig. 3 EELS identification of distinct electronic states in the epi-SLs. (a) HAADF reference image of a region with a single isolated QD in proximity to an area of epi-SL. (b) HAADF reference image from the SI acquired from the green highlighted area in (a). (c) Three representative spectra from different regions in the SI representing the substrate signal $\left(\mathrm{SiO}_{2}\right)$, an isolated PbSe QD (QD), and its epi-SL, the pixels used for the representative spectra are labeled in (b). (d) The difference spectra taken from the spectra in (c). The QD-SiO 2 difference spectrum represents the low-loss signal from the PbSe QD states (which begin at the nominal $0.7 \mathrm{eV}$ band gap of the $6.5 \mathrm{~nm}$ diameter PbSe QDs), and the SL-QD difference spectrum represents the new states generated by the epitaxial connectivity in the necks (which show a sharp onset in intensity at $1.1 \mathrm{eV}$ band gap of the $3.5 \mathrm{~nm}$ diameter necks). 
To demonstrate the repeatability of the new states emerging from the neck and their localization, various regions of the samples are studied with three component non-negative matrix factorization (NMF) decomposition of the SI presented in Fig. 4a-c. The representative spectra from the marked regions in Fig. 4a-c presented in Fig. 4d are similar, especially concerning the $1.1 \mathrm{eV}$ onset, indicating similar electronic structure. Three component NMF decomposition analysis was performed for all three datasets which are shown in Fig. 4e-p. Interestingly, the NMF decomposition returns three components. The first component captures the tail of the zero-loss peak (ZLP) and the $\mathrm{SiO}_{2}$ substrate. The second component captures the behavior of isolated $\mathrm{PbSe}$ QDs (which is referred as the 'bulk' response). The third component captures the new electronic states in the epi-SL.

The abundance maps of the first NMF component (ZLP and substrate) are presented in Fig. 4e-g which show uniform contrast corresponding to the uniform beam condition and membrane. The spectral endmembers are plotted in Fig. 4h, which matches well with the $\mathrm{SiO}_{2}$ representative spectra from Fig. 3. The abundance maps of the second component (bulk response) are shown in Fig. 4i-k, and they show that these bulk PbSe states are observed everywhere regardless of the presence of epitaxial necking. Furthermore, in Fig. 4k the spectral endmembers of the NMF components match the QD-SiO 2 difference spectrum, confirming that this component represents the bulk response of PbSe QDs. Lastly, the maps of the third component (new electronic states induced by the epi-SL) are shown in Fig. 4m-o. In datasets 1 and 2, the electronic states corresponding to $1.1 \mathrm{eV}$ are visible in epi-SL while the isolated QD in Fig. 4m and the trimer in Fig. 4 do not light up indicating the present of this component requires QD necking. In dataset 3 (Fig. 4o) there is only a single isolated QD in the top right corner, and this QD also shows no intensity of the epi-SL states. More importantly, the spectral endmembers of all three NMF 
decompositions in Fig. $4 p$ show the exact same spectral form as the SL-QD difference spectrum from Fig. 3, demonstrating that this component is effectively a map of the new epi-SL electronic states. Furthermore, it illustrates that the EEL intensity in spectral regime is a fundamental feature of the electronic structure in epi-SL, as other collective phenomena (such as plasmons and polaritons) would be highly geometry-dependent and vary in frequency and linewidth as a function of the epi-SL shape.

One of the most notable aspects of the epi-SL component, in the large-area dataset 3 , is the presence of dead spots where the epi-SL states are significantly suppressed compared to the surrounding regions. Furthermore, while some of these dead spots occur in areas that exhibit irregular structure in the corresponding HAADF of Figure 4c, some appear in places that are nominally uniform. This implies that poor necking formation can influence the formation of these new electronic states, but also perhaps deeper atomistic structural effects can play an important role as well. The other important aspect to note here, is that significant intensity is observed outside of the epi-SL itself. This phenomenon is called the 'aloof' effect which allows the evanescent field of the electrons to interact with the sample non-locally, causing significant intensity outside of the $\mathrm{PbSe}$. The aloof interaction can be cut-out with a technique called Off-Axis EELS to show that the signal is totally localized to the epi-SL, which is demonstrated in the Fig. S7. 


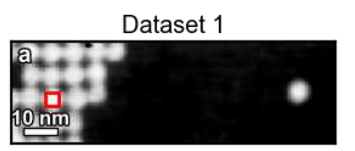

Dataset 2
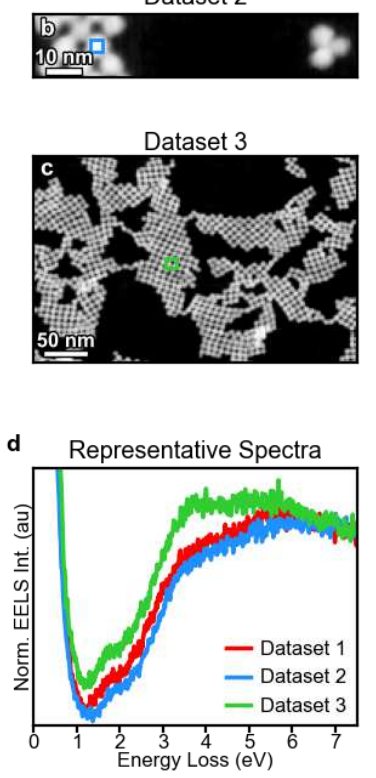
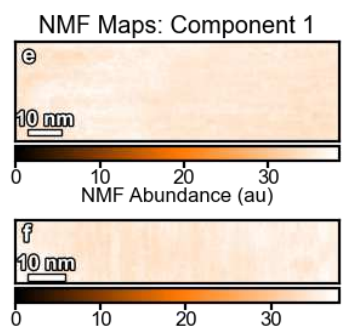

NMF Abundance (au)

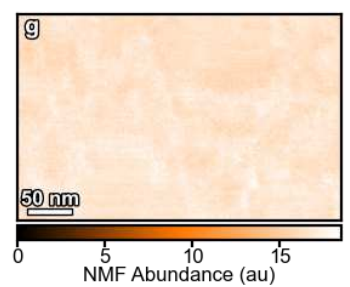

h NMF Spectra: Component 1

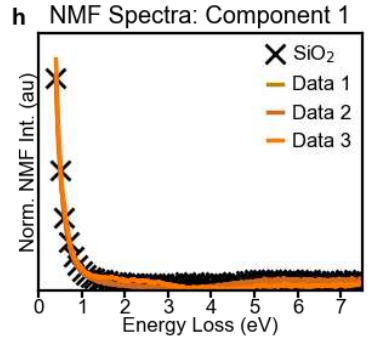

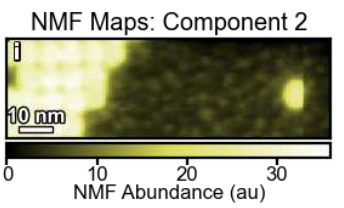
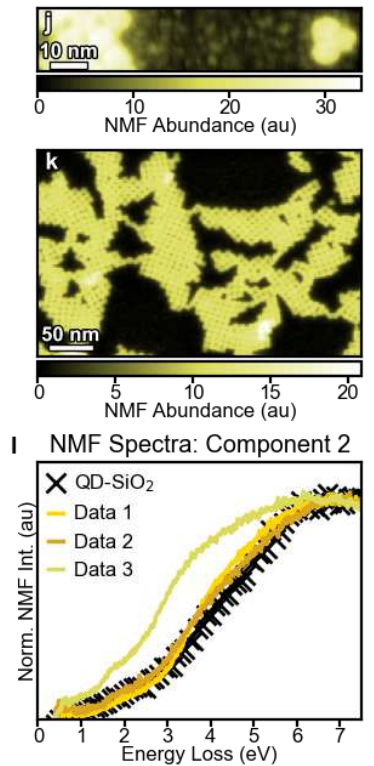
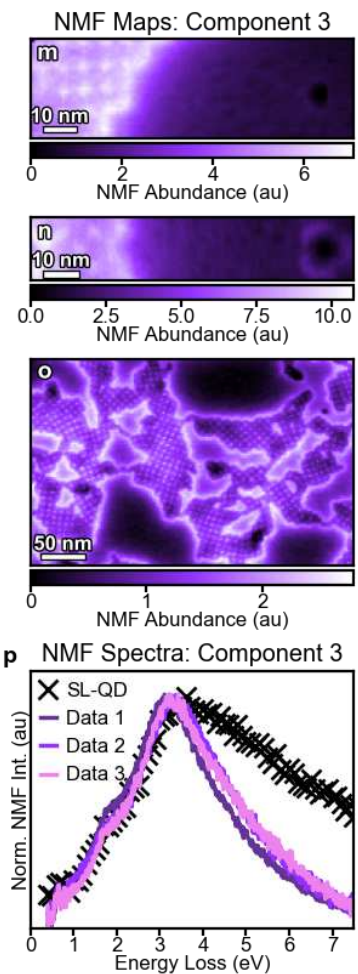

Fig. 4 Realization of Band Gap Modulation in epi-SLs in various regions with NMF analysis.

Three EEL SIs of QDs with no necks compared to the epi-SLs with necking. (a-c) HAADF STEM reference images of the three datasets. (a) shows the dataset with only a single isolated QD located nearby epi-SL from the fig. 3. (b) shows a trimer cluster without necking, (c) shows a large area epi-SL containing an isolated QDs and various necking configurations. (d) Shows the representative spectra from the marked regions in (a), (b), and (c) showing that the electronic structures of the three epi-SLs is nearly identical. NMF decomposition and visual representation of the SI shown in (e-p). (e-g), (i-k) and (m-o) presents the abundance maps of showing the localization of the substrate signal, PbSe QD states and epi-SL states from region in (a), (b) and (c) respectively. (h), (l), (p) shows the NMF spectral endmembers correspondingly. The maps show that new electronic states are generated by the epitaxial connections in the epi-SL. From this it can be clearly seen that the trends of the necking in the epi-SL inducing new electronic states (component 3) at low energies is repeatable. These indicate that the agglomeration of the QDs will not induce these new electronic states (component 3) instead the necking induces the new electronic states. 


\section{Conclusion}

Direct measurements of local electronic structure of PbSe QD epi-SLs are reported. The structural analysis with STEM-HAADF confirms the presence of epitaxial necks between QDs in the epiSL structure. However, as reported previously, variation in QD and neck size as well as missing QDs and necks induce structural disorder and variation in the electronic structure of the epi-SL. STS analysis revealed modulation of the band gap from $0.7 \mathrm{eV} \pm 0.05$ in the QDs to $1.1 \pm 0.02 \mathrm{eV}$ in the necks. Distinct electronic states induced by the necks were also confirmed by EELS measurements. Decomposition of the EELS data with NMF analysis for a comparatively largearea epi-SL further confirmed the existence of distinct electronic states within the epi-SL at the necks. Associating the observed large band gap of $1.1 \mathrm{eV}$ at the neck with its $3.5 \mathrm{~nm}$ effective diameter resembles the quantum confinement effect in a $\mathrm{QD}$, for which the band gap is inversely proportional to QD size. This suggests the emergence of new electronic states induced by quantum confinement of carriers within the epi-SL necks, showing the distinct electronic structure in the neck and how it shapes the energy landscape for charge carriers moving through the epi-SL. This result also implies that there is coherence between the QDs in the epi-SL, but nonuniformity of the QD and neck sizes as well as aperiodicity in the lattice induce significant disorder across the epiSL, hindering the observation of coherent states at the mesoscale. 


\section{Methods}

Materials. All chemicals were used as received unless otherwise noted. Lead oxide (PbO, 99.999\%), lead iodide $\left(\mathrm{PbI}_{2}, 99.9985 \%\right)$ and selenium shot (99.999\%) were purchased from Alfa Aesar. Oleic acid (OA, technical grade, 90\%), diphenylphosphine (DPP, 98\%), 1-octadecene (ODE, 90\%), anhydrous ethylene glycol (EG, 99.8\%), anhydrous acetonitrile (99.99\%), anhydrous hexanes (99\%), anhydrous toluene (99.8\%), 3-mercaptopropyltrimethoxysilane (3-MPTMS, 95\%), trimethylaluminum (TMA, 97\%), ammonium thiocyanate $\left(\mathrm{NH}_{4} \mathrm{SCN}, 99.99 \%\right)$ and anhydrous dimethyl sulfoxide (DMSO, 99.9\%) were purchased from Sigma Aldrich. Anhydrous 1,2-ethylenediamine (EDA, >98.0\%) was purchased from TCI. Trioctylphosphine (TOP, technical grade, $>90 \%$ ) was acquired from Fluka and mixed with selenium shot for 24 hours to form a $1 \mathrm{M}$ TOP-Se stock solution. 18.2 M $\Omega$ water (Milli-Q Gradient) was used for substrate cleaning and atomic layer deposition (ALD). Water for ALD was degassed with three freeze-pump-thaw cycles before use.

Quantum dot synthesis. PbSe QDs were synthesized and purified using standard air-free techniques according to a previously published procedure ${ }^{10}$. Briefly, $\mathrm{PbO}(1.50 \mathrm{~g}), \mathrm{OA}(5.00 \mathrm{~g})$, and ODE (10.00 g) were mixed and degassed in a three-neck round-bottom flask at room temperature. The mixture was then heated at $120{ }^{\circ} \mathrm{C}$ under vacuum to form $\mathrm{Pb}(\mathrm{OA})_{2}$ and dry the solution. After 1.5 hours, the $\mathrm{Pb}(\mathrm{OA})_{2}$ solution was heated to $180{ }^{\circ} \mathrm{C}$ under argon flow and $9.5 \mathrm{~mL}$ of a 1 M solution of TOP-Se containing $200 \mu \mathrm{L}$ of DPP was rapidly injected into this hot solution. An immediate darkening of the solution was observed, and the QDs were grown for 105 seconds at $\sim 160{ }^{\circ} \mathrm{C}$. The reaction was quenched with a liquid nitrogen bath and injection of $10 \mathrm{~mL}$ of anhydrous hexanes. The QDs were purified in an $\mathrm{N}_{2}$-filled glovebox $\left(<0.5 \mathrm{ppm} \mathrm{O}_{2}\right)$ by adding 2 $\mathrm{mL}$ of toluene and $9 \mathrm{~mL}$ of acetonitrile to the reaction solution, collecting the QDs by 
centrifugation, performing 6-8 cycles of redispersion/precipitation using toluene/acetonitrile (3 $\mathrm{mL} / 24 \mathrm{~mL}$ ), and then drying and storing the QDs as a powder in the glovebox.

Fabrication of multilayer QD superlattices for STM/STS experiments. Multilayer (3D) epi-SL films consisting of 6-7 QD layers ( 40 nm thick) were made using a modified version of a published procedure ${ }^{10}$. Briefly, in an $\mathrm{N}_{2}$-filled glovebox with $<0.5 \mathrm{ppm} \mathrm{O}_{2}$, oleate-capped $\mathrm{PbSe}$ QD superlattices were prepared by carefully drop casting $60 \mu \mathrm{L}$ of a $15 \mathrm{mg} / \mathrm{mL}$ solution of PbSe QDs dispersed in hexanes onto $7 \mathrm{~mL}$ of ethylene glycol in a Teflon well $(3.5 \mathrm{~cm}$ wide $\times 5 \mathrm{~cm}$ long $\times 1 \mathrm{~cm}$ deep) that was cleaned by soaking overnight in $5 \mathrm{M}$ nitric acid and then soaking and rinsing in Millipore water at least ten times. After depositing the QD solution, the well was immediately covered with a glass slide and the hexanes allowed to slowly evaporate in 25-30 minutes, resulting in a smooth, dry QD film floating on the EG surface. The slide was then removed and $100 \mu \mathrm{L}$ of a 7.5 M solution of ethylenediamine in acetonitrile was slowly (5-10 s) injected into the EG directly underneath a corner of the film. As the EDA solution spread throughout the well, the film visibly darkened, indicating film densification and conversion to the epi-SL. After 30 seconds, the darkened epi-SL film nearest to the EDA injection point was transferred onto $\mathrm{SiO}_{2} / \mathrm{Si}(30 \mathrm{~nm}$ thermal $\mathrm{SiO}_{2}$ ) substrate by manual stamping using a vacuum wand. Prior to stamping, the $\mathrm{SiO}_{2}$ coated Si substrate was cleaned by 15 minutes of sonication in acetone, water, and isopropanol, dried under flowing air, soaked in a $100 \mathrm{mM}$ solution of 3-MPTMS in toluene for 1 hour to functionalize the surface for improved QD adhesion, then rinsed with neat toluene and dried under flowing air. The stamped film was rinsed vigorously with neat acetonitrile and dried under flowing $\mathrm{N}_{2}$. Next, the epi-SL film was immediately soaked in a $10 \mathrm{mM}$ solution of $\mathrm{PbI}_{2}$ in DMSO for 5 minutes to reduce the amount of residual oleate and adsorbed glycoxide, rinsed with copious amounts of DMSO and acetonitrile, and dried under flowing $\mathrm{N}_{2}$. 
Fabrication of monolayer QD superlattices for STEM EELS experiments. Monolayer (2D) epi-

SL films for EELS experiments were made as follows. An aliquot of a $1.75 \mathrm{mg} / \mathrm{mL} \mathrm{PbSe} \mathrm{QD}$ dispersion in toluene was drop cast onto a Pelco $\mathrm{SiO}_{2}$ support film TEM grid ( $8 \mathrm{~nm}$ thick $\mathrm{SiO}_{2}$ windows, product 21532-10, Ted Pella) and dried over the course of several minutes. The grid was then immersed for 15 seconds in a $15 \mathrm{mM}$ ammonium thiocyanate $\left(\mathrm{NH}_{4} \mathrm{SCN}\right)$ solution in acetonitrile, soaked in neat acetonitrile for 10 seconds to remove unbound $\mathrm{NH}_{4} \mathrm{SCN}$, dried, soaked in neat hexanes for 10 seconds to remove residual unbound oleic acid/oleate, dried again, and finally dipped into neat acetonitrile, quickly removed, and blown dry in a stream of $\mathrm{N}_{2}$ to prevent solvent droplets from depositing residue onto the grid surface.

STM-STS Analysis. A cryogenic four-probe scanning tunneling microscope (4P-STM) from RHK/UNISOKU at ORNL was used to measure in situ two-probe STM/STS in multilayer epiSLs. Measurements were performed in UHV $\left(5 \times 10^{-10}\right.$ Torr) at both $82 \mathrm{~K}$ and $300 \mathrm{~K}$. Epi-SL samples were transferred from a glovebox $\left(<0.1 \mathrm{ppm} \mathrm{O}_{2}\right.$ and $\left.<1 \mathrm{ppm}_{2} \mathrm{O}\right)$ to the UHV chamber using a nitrogen-filled bag and a small glovebox that enclosed the load-lock. In situ SEM imaging in the STM was used to identify epi-SL grains and precisely locate the STM probe tips on the sample. For STM/STS measurements, one of the probes was pressed a few nm into the sample to make a good electrical contact to the epi-SL and then the bias voltage was applied, while the other probe was used to scan the sample with tunneling current kept constant with the feedback loop. Conventional lock-in techniques were used to measure the $\mathrm{d} / / \mathrm{d} V$ spectra, where the modulation frequency was $1 \mathrm{kHz}$ and the modulation voltage $30 \mathrm{mV}$. The energy conversion from the tip-bias voltage applied in STS measurements was obtained with correction factor of 0.8 (from tip-induced band banding) derived previously ${ }^{14}$. In this consideration, the QD below the tip is nearly under the 
same potential with scanning probe since $\mathrm{PbSe} \mathrm{QD}$ has a large dielectric constant ( $\mathrm{OD}=4 \mathrm{~nm}, \varepsilon$ =227). Therefore, the lever arm, $\eta=V_{\mathrm{t}} / V$ fraction is close to unity ${ }^{14}$.

STEM-EELS Analysis. STEM-EELS experiments were performed on the monolayer epi-SL samples in a double aberration corrected, monochromated Nion Ultra-HERMES STEM microscope $\mathrm{e}^{29}$ equipped with a Nion Iris spectrometer operating at $60 \mathrm{keV}$ and an energy resolution of $100 \mathrm{meV}$ at Oak Ridge National Laboratory. This energy resolution was selected as the best balance of resolution and signal (beam current). EEL spectra were acquired using a $\sim 60 \mathrm{pA}$ beam current and a convergence angle of $30 \mathrm{mrad}$ through a $1 \mathrm{~mm}$ aperture, corresponding to a $25 \mathrm{mrad}$ collection angle. Multiple EEL spectra were acquired and summed to a single spectrum using subpixel alignment to maximize the signal-to-noise ratio. A post-processing normalization routine was used to account for the significant difference in the inelastic signal when the probe was passing through the QDs vs. only the substrate (See Figure S6). Cherenkov radiation resulting from electrons having velocities greater speed than the speed of light in the material, is a serious concern for this type of low-loss analysis but it is avoided by using a $60 \mathrm{keV}$ electron beam, as reported previously ${ }^{26,27}$. 
Competing Interest: The authors declare no competing interests.

Additional Information: Supplementary information is available for this paper.

Author contributions: M.S.K. initiated the project, planned, and conducted the research under the supervision of M.L. Samples were prepared by C.Q. and A.A. and samples with assistance from M.S.K. and H.K. M.S.K. and E.B.U. developed an algorithm and performed structural analysis using STEM images. W.K. and M.S.K. conducted STM-STS experiments supervised by A.P.L. J.H. and M.S.K. performed STEM-EELS experiment and analyzed the data with J.C.I. All authors contributed to the interpretation of the results. M.S.K., J.H., and W.K. drafted the manuscript. All authors reviewed and commented on the manuscript.

Acknowledgements. This work was supported by the UC Office of the President under the UC Laboratory Fees Research Program Collaborative Research and Training Award LFR-17-477148. The authors acknowledge the use of facilities and instrumentation at the UC Irvine Materials Research Institute (IMRI), which is supported in part by the National Science Foundation through the UC Irvine Materials Research Science and Engineering Center (DMR-2011967). STM-STS and STEM-EELS were conducted at the Center for Nanophase Materials Sciences, which is a US Department of Energy Office of Science User Facility. Microscopy performed using instrumentation within ORNL's Materials Characterization Core provided by UT-Battelle, LLC, under Contract No. DE-AC05- 00OR22725 with the DOE and sponsored by the Laboratory Directed Research and Development Program of Oak Ridge National Laboratory, managed by UT-Battelle, LLC, for the U.S. Department of Energy 


\section{References}

1 Brus, L. E. Electron-electron and electron-hole interactions in small semiconductor crystallites: The size dependence of the lowest excited electronic state. The Journal of Chemical Physics 80, 4403-4409, doi:10.1063/1.447218 (1984).

2 Alivisatos, A. P. Semiconductor Clusters, Nanocrystals, and Quantum Dots. Science 271, 933-937, doi:10.1126/science.271.5251.933 (1996).

3 Ashoori, R. C. Electrons in artificial atoms. Nature 379, 413-419, doi:10.1038/379413a0 (1996).

4 Banin, U., Cao, Y., Katz, D. \& Millo, O. Identification of atomic-like electronic states in indium arsenide nanocrystal quantum dots. Nature 400, 542-544, doi:10.1038/22979 (1999).

5 Dekorsy, T., Ott, R., Kurz, H. \& Köhler, K. Bloch oscillations at room temperature. Physical Review B 51, 17275-17278, doi:10.1103/PhysRevB.51.17275 (1995).

6 Leo, K., Bolivar, P. H., Brüggemann, F., Schwedler, R. \& Köhler, K. Observation of Bloch oscillations in a semiconductor superlattice. Solid State Communications 84, 943946, doi:https://doi.org/10.1016/0038-1098(92)90798-E (1992).

7 Whitham, K. et al. Charge transport and localization in atomically coherent quantum dot solids. Nature Materials 15, 557-563, doi:10.1038/nmat4576 (2016).

8 Boles, M. A., Engel, M. \& Talapin, D. V. Self-Assembly of Colloidal Nanocrystals: From Intricate Structures to Functional Materials. Chemical Reviews 116, 11220-11289, doi:10.1021/acs.chemrev.6b00196 (2016).

9 Dalven, R. A review of the semiconductor properties of $\mathrm{PbTe}, \mathrm{PbSe}, \mathrm{PbS}$ and $\mathrm{PbO}$. Infrared Physics 9, 141-184, doi:https://doi.org/10.1016/0020-0891(69)90022-0 (1969).

10 Abelson, A. et al. Collective topo-epitaxy in the self-assembly of a 3D quantum dot superlattice. Nature Materials 19, 49-55, doi:10.1038/s41563-019-0485-2 (2020).

11 Chu, X. et al. Structural characterization of a polycrystalline epitaxially-fused colloidal quantum dot superlattice by electron tomography. Journal of Materials Chemistry A $\mathbf{8}$, 18254-18265, doi:10.1039/D0TA06704K (2020).

12 Moreels, I. et al. Composition and Size-Dependent Extinction Coefficient of Colloidal PbSe Quantum Dots. Chemistry of Materials 19, 6101-6106, doi:10.1021/cm071410q (2007).

13 Tersoff, J. \& Hamann, D. R. Theory of the scanning tunneling microscope. Physical Review B 31, 805-813, doi:10.1103/PhysRevB.31.805 (1985).

14 Swart, I., Liljeroth, P. \& Vanmaekelbergh, D. Scanning probe microscopy and spectroscopy of colloidal semiconductor nanocrystals and assembled structures. Chemical Reviews 116, 11181-11219, doi:10.1021/acs.chemrev.5b00678 (2016).

15 Liljeroth, P. et al. Density of States Measured by Scanning-Tunneling Spectroscopy Sheds New Light on the Optical Transitions in PbSe Nanocrystals. Physical Review Letters 95, 086801, doi:10.1103/PhysRevLett.95.086801 (2005).

16 Ueda, S. T. et al. Electronic passivation of PbSe quantum dot solids by trimethylaluminum vapor dosing. Applied Surface Science 513, 145812, doi:https://doi.org/10.1016/j.apsusc.2020.145812 (2020).

17 Capiod, P. et al. Electronic properties of atomically coherent square PbSe nanocrystal superlattice resolved by Scanning Tunneling Spectroscopy. Nanotechnology 32, 325706, doi:10.1088/1361-6528/abfd57 (2021). 
18 Kolmer, M. et al. Electronic transport in planar atomic-scale structures measured by twoprobe scanning tunneling spectroscopy. Nature Communications 10, 1573, doi:10.1038/s41467-019-09315-6 (2019).

19 Nguyen, G. D. et al. Atomically Precise PdSe2 Pentagonal Nanoribbons. ACS Nano 14, 1951-1957, doi:10.1021/acsnano.9b08390 (2020).

20 Bakkers, E. P. A. M. et al. Shell-Tunneling Spectroscopy of the Single-Particle Energy Levels of Insulating Quantum Dots. Nano Letters 1, 551-556, doi:10.1021/n1015572b (2001).

21 Liljeroth, P. et al. Variable Orbital Coupling in a Two-Dimensional Quantum-Dot Solid Probed on a Local Scale. Physical Review Letters 97, 096803, doi:10.1103/PhysRevLett.97.096803 (2006).

22 Overgaag, K., Liljeroth, P., Grandidier, B. \& Vanmaekelbergh, D. Scanning Tunneling Spectroscopy of Individual PbSe Quantum Dots and Molecular Aggregates Stabilized in an Inert Nanocrystal Matrix. ACS Nano 2, 600-606, doi:10.1021/nn7003876 (2008).

23 Jdira, L. et al. Linewidth of resonances in scanning tunneling spectroscopy. Physical Review B 77, 205308, doi:10.1103/PhysRevB.77.205308 (2008).

24 Klein, M. \& Schwitzgebel, G. An improved lamellae drop-off technique for sharp tip preparation in scanning tunneling microscopy. Review of Scientific Instruments 68, 30993103, doi:10.1063/1.1148249 (1997).

25 Müller, A. D. et al. Characterization of electrochemically etched tungsten tips for scanning tunneling microscopy. Review of Scientific Instruments 70, 3970-3972, doi:10.1063/1.1150022 (1999).

26 Jung, H. J. et al. Spatial Variation of Available Electronic Excitations within Individual Quantum Dots. Nano Letters 13, 716-721, doi:10.1021/nl304400c (2013).

27 Logar, M., Xu, S., Acharya, S. \& Prinz, F. B. Variation of Energy Density of States in Quantum Dot Arrays due to Interparticle Electronic Coupling. Nano Letters 15, 18551860, doi:10.1021/nl5046507 (2015).

$28 \mathrm{Gu}$, L. et al. Band-gap measurements of direct and indirect semiconductors using monochromated electrons. Physical Review B 75, 195214, doi:10.1103/PhysRevB.75.195214 (2007).

29 Hachtel, J. A., Lupini, A. R. \& Idrobo, J. C. Exploring the capabilities of monochromated electron energy loss spectroscopy in the infrared regime. Scientific Reports 8, 5637, doi:10.1038/s41598-018-23805-5 (2018). 


\section{Supplementary Files}

This is a list of supplementary files associated with this preprint. Click to download.

- SIEmergenceofDistinctElectronicStatesinEpitaxiallyFusedPbSeQuantumDotSuperlatticessubmitted.pdf 